\title{
SPECTRAL DECOMPOSITION OF DISPERSION MATRIX FOR THE MIXED ANALYSIS OF VARIANCE MODEL
}

\author{
B. GÜVEN
}

Department of Statistics, Middle East Technical University 06531, Ankara Turkey.

(Received June 27, 2000; Accepted Oct. 11, 2000)

\begin{abstract}
The spectral decomposition of the variance-covariance matrix for a balanced mixed analysis of variance model is presented. The model consists of crossed and/or nested factors with either replicated or nonreplicated.
\end{abstract}

\section{INTRODUCTION}

The spectral decomposition of a variance-covariance matrix (dispersion matrix) $\mathbf{V}$ is useful for finding its powers $\mathbf{V}^{\alpha}$ where $\alpha$ is any real number. In particular, $\alpha=-1, V^{-1}$ is useful for estimation or $\alpha=-\frac{1}{2}, V^{-1 / 2}$ is useful for the transforming a linear model to a model with i.i.d. error terms.

The problem has been discussed before by Searle and Henderson [1] and Wansbeek and Kapteyn [2]. In both studies, it is supposed that the form of the spectral decomposition of $\mathbf{V}$ is of the same form of $\mathbf{V}$. Then they obtained idempotent matrices in the spectral decomposition of $\mathbf{V}$ by equating $\mathbf{V}$ and its assumed spectral decomposition.

However our solution is based on deriving an idempotent matrix from eigenvectors for the corresponding eigenvalue in the spectral decomposition of $\mathbf{V}$ without assuming any form of the spectral decomposition of $\mathbf{V}$.

\section{THE DISPERSION MATRIX}

The variance-covariance matrix $\mathbf{V}$ for a balanced $\mathrm{k}$-factor mixed analysis of variance model is of the following structure

$$
\mathbf{V}=\sum_{\mathbf{d}} \boldsymbol{\lambda}_{\mathbf{d}} \mathbf{N}_{\mathbf{d}}
$$


where $\mathbf{d}$ is a $\mathrm{k}$-vector of zeros and ones. The summation is taken over $2^{\mathrm{k}}$-elements. The $\lambda_{d}$ are nonnegative parameters. Let $d=\left(i_{1}, i_{2}, \ldots, i_{k}\right)$ with $i_{r}$ or 1 for $t=1,2 \ldots k$. Then the matrix $\mathbf{N}_{\mathbf{d}}$ in (1) are defined as

$$
\mathbf{N}_{\mathbf{d}}=\mathbf{J}_{1}^{\mathrm{i}_{1}} \otimes \mathbf{J}_{2}^{\mathrm{i}_{2}} \otimes \ldots \otimes \mathbf{J}_{\mathbf{k}}^{\mathrm{i}_{\mathbf{k}}}
$$

with $\mathbf{J}_{\mathbf{x}}^{0}=\mathbf{I}_{\mathrm{r}}$, where $\mathbf{J}_{\mathrm{x}}$ and $\mathbf{I}_{\mathrm{x}}$ are respectively a matrix of ones and an identity matrix of order $\mathrm{n}_{\mathrm{r}}$ for $\mathrm{r}=1,2, \ldots, \mathrm{k}$, the symbol $\otimes$ denotes the Kronecker product of matrices.

The full rank of (1), leading that all eigenvalues of (1) are nonzero, is provided by $\mathbf{N}_{0 \theta \ldots 00}=\mathbf{I}_{12 \ldots k}$ where $\mathbf{I}_{12 \ldots . . \mathrm{k}}$ is an identity matrix of order $\prod_{r=1}^{k} \mathrm{n}_{\mathrm{r}}$. A linear space generated by the columns of (1) is the sum of linear subspaces generated by the columns of $2^{k}$ matrices of order $\prod_{r=1}^{\mathbb{k}} n_{r}$ given by $I_{12 \ldots k}, N_{00} \ldots 01, \ldots, N_{11 \ldots 11}$ and then is spanned by the set of basis $I_{12 \ldots k}, N_{00 \ldots 01} \ldots, N_{11 \ldots 11}$. However the basis for any $\mathbf{N}_{\mathrm{i}_{1} \mathbf{i}_{2} \ldots \mathrm{i}_{\mathbf{k}}}$ are the linear combination of the basis for $\mathbf{I}_{12 \ldots \mathbf{k}}$. As a result, a linear space generated by the columns of (1) is spanned by the set of basis for $\mathbf{I}_{12 \ldots \mathrm{k}}$.

\section{THE SPECTRAL DECOMPOSIITION}

Let $t_{1}, t_{2}, \ldots, t_{k}$ be denoted by $t$ with $t_{r}=0$ or 1 . The $2^{k}$ (possibly) distinct eigenvalues of (1) given by [1] are:

$$
\phi_{\mathbf{t}}=\sum_{\mathbf{d}} \lambda_{\mathbf{d}} \mathbf{x}_{\mathbf{t}_{1}}^{\mathbf{i}_{1}} \mathbf{x}_{\mathbf{t}_{2}}^{\mathbf{i}_{2}} \ldots \mathbf{x}_{\mathbf{t}_{\mathbf{k}}}^{\mathbf{i}_{\mathbf{k}}}
$$

with multiplicity $\prod_{r=1}^{k}\left(n_{r}-1\right)^{1-t_{r}}$ and where $x_{t_{r}}^{i_{r}}$ is the eigenvalue of the matrix $J_{x}^{i_{r}}$ given by

$$
x_{t_{r}}^{i_{r}}=\left\{\begin{array}{lll}
0 & \text { if } & t_{r}=0 \\
n_{r} & \text { if } & t_{r}=1
\end{array}\right.
$$

with multiplicity $\left(n_{r}-1\right)^{1-t_{r}}$ if $i_{r}=1 . x_{t_{r}}^{i_{r}}=1$ for $t_{r}=0,1$ with multiplicity $n_{r}$ if $i_{r}=0$. An eigenvector $v_{t_{\mathrm{r}}}$ for $x_{t_{\mathrm{r}}}^{i_{\mathrm{r}}}$ will be:

$$
\mathbf{v}_{t_{r}}=\left\{\begin{array}{llll}
\xi_{n_{r} k} & k=1,2, \ldots, n_{r}-1 & \text { if } & t_{r}=0 \\
\frac{1}{\sqrt{n_{r}}} \mathbf{l}_{r} & \text { if } & t_{r}=1
\end{array}\right.
$$

and $\xi_{n_{r}}, \xi_{n_{r}}, \ldots, \xi_{n_{r} \mathbf{u}_{r}-1}, \frac{1}{\sqrt{n_{r}}} 1_{r}$ is an orthonormal set, $1_{r}$ is a $n_{r} \times 1$ vector of ones.

Let

$$
v_{t}=v_{t_{1}} \otimes v_{t_{2}} \otimes \ldots \otimes v_{t_{k}}
$$


Then $v_{t}$ is an eigenvector for $\phi_{d}$ in (2) since ${J_{r}}_{i_{r}}^{i_{r}} v_{t_{r}}=x_{t_{r}}^{i_{r}} v_{t_{T}}$ and

$$
\begin{aligned}
& \mathbf{N}_{\mathbf{d}} \boldsymbol{v}_{\mathbf{t}}=\left(\mathbf{J}_{1}^{\mathbf{i}_{1}} \otimes \mathbf{J}_{2}^{\mathbf{i}_{2}} \otimes \ldots \otimes \mathbf{J}_{\mathrm{k}}^{\mathbf{i}_{\mathrm{k}}}\right)\left(\mathbf{v}_{\mathbf{t}_{1}} \otimes \mathbf{v}_{\mathbf{t}_{2}} \otimes \ldots \otimes \mathbf{v}_{\mathbf{t}_{\mathrm{k}}}\right) \\
& =\mathbf{J}_{1}^{i_{1}} \mathbf{v}_{\mathbf{t}_{1}} \otimes \mathbf{J}_{2}^{i_{2}} \mathbf{v}_{\mathbf{t}_{2}} \otimes \ldots \otimes \mathbf{J}_{\mathbf{k}}^{i_{k}} \mathbf{v}_{\mathbf{t}_{k}} \\
& =x_{t_{1}}^{i_{1}} \mathbf{v}_{t_{1}} \otimes x_{t_{2}}^{i_{z}} \mathbf{v}_{t_{2}} \otimes \ldots \otimes x_{t_{k}}^{i_{k}} v_{t_{k}}=\left(x_{t_{1}}^{i_{1}} x_{t_{2}}^{i_{z}} \ldots x_{t_{k}}^{i_{k}}\right)\left(v_{t_{1}} \otimes v_{t_{2}} \otimes \ldots \otimes v_{t_{k}}\right) .
\end{aligned}
$$

Consequently,

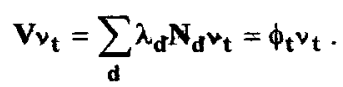

Let $\mathbf{P}_{\mathrm{t}_{\mathrm{T}}}=\mathbf{v}_{\mathrm{t}_{\mathrm{T}}} \mathbf{v}_{\mathrm{t}_{\mathrm{T}}}^{\prime}$ for $\mathrm{r}=1,2, \ldots, \mathrm{k}$ where $\mathbf{v}_{\mathbf{t}_{\mathrm{T}}}$ in (3) is an eigenvector of $\mathbf{J}_{\mathrm{r}}^{\mathrm{i}_{\mathrm{r}}}$ and $\mathbf{M}_{t}=v_{t} v_{t}^{\prime}$ where $v_{t}$ in (4) is an eigenvector of (1). Then both $P_{t_{r}}$ and $M_{t}$ are idempotent matrices and the spectral decomposition of $(1)$ is:

$$
\mathbf{v}=\sum_{t} \phi_{t} \mathbf{M}_{t}
$$

where

$$
\mathbf{M}_{\mathbf{t}}=\mathbf{P}_{\mathbf{t}_{1}} \otimes \mathbf{P}_{\mathbf{t}_{2}} \otimes \ldots \otimes \mathbf{P}_{\mathbf{t}_{\mathrm{r}}}
$$

with

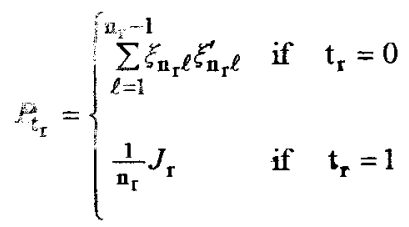

Corsider a matrix $I_{r}+J_{r}$ having eigenvalues 1 with multiplicity $n_{r}-1$ and $1+\mathrm{n}_{\mathrm{z}_{\mathrm{r}}}$ and the respective orthonormal eigenvectors $\xi_{\mathrm{n}_{\mathrm{r}} \ell}, \ell=1,2, \ldots, \mathrm{n}_{\mathrm{r}}-1$ for 1 and $\sqrt{m_{\mathrm{r}}} \mathbf{i}_{\mathrm{r}}$. Then the spectral decomposition of $\mathbf{I}_{\mathrm{r}}+\mathbf{J}_{\mathrm{i}}$ is:

$$
\mathbf{I}_{\mathrm{r}}+\mathbf{J}_{\mathrm{r}}=\sum_{\ell=1}^{n_{\mathrm{r}}-1} \xi_{\mathrm{n}_{\mathrm{r}}} \xi_{\mathrm{n}_{\mathrm{r}} \ell}+\left(1+\mathrm{n}_{\mathrm{r}}\right) \frac{1}{\mathbf{n}_{\mathrm{r}}} J_{\mathrm{r}}
$$

Using (7), (6) can be rewritten as:

$$
\mathbf{P}_{\mathbf{t}_{\mathrm{r}}}=\left\{\begin{array}{lll}
\mathbf{I}_{\mathbf{r}}-\frac{1}{\mathbf{n}_{\mathrm{r}}} \mathbf{J}_{\mathbf{r}} & \text { if } & t_{\mathbf{r}}=0 \\
\frac{1}{\mathbf{n}_{\mathrm{r}}} \mathbf{J}_{\mathbf{r}} & \text { if } & \mathbf{t}_{\mathbf{r}}=1
\end{array}\right.
$$

where the rank of $P_{t_{r}}$ is $\left(n_{x}-1\right)^{1-t_{T}}$. From (5) with (8), it can be seen that $\mathbf{M}_{t}$ has $\operatorname{rank} \sum_{r=1}^{k}\left(n_{k}-1\right)^{1-t_{r}}$ and $M_{t} M_{t^{*}}=0$ for $t \neq t^{*}$.

Consider a mixed model representing an experiment that is replicated $n_{k}$-times. (1) can be rewritten as 


$$
\mathbf{V}=\lambda_{00 \ldots 00} \mathbf{I}_{\mathbf{n}}+\sum_{\mathbf{d}} \lambda_{\mathbf{d}} \mathbf{N}_{\mathbf{d}}
$$

since $\lambda_{00 \ldots 00}$ is positive and $\lambda_{t_{1} t_{2} \ldots t_{k-1} 0}$ is zero for at least one of nonzero $t_{r}$ where $r=1,2, \ldots, k-1$. Here $d=\left(i_{1} i_{2} \ldots i_{k-1}\right)$ with $i_{i}=0$ for $r=0,1, \ldots k-1$, the summation on the right hand side of (9) is taken over $2^{k-1}$-elements and

It follows that (2) can be:

$$
\mathbf{N}_{\mathbf{d}}=\mathbf{J}_{1}^{\mathbf{i}_{1}} \otimes \mathbf{J}_{2}^{\mathrm{i}_{2}} \otimes \ldots \otimes \mathbf{J}_{\mathbf{k}-1}^{\mathbf{i}_{\mathbf{k}}-1} \otimes \mathbf{J}_{\mathbf{k}}
$$

$$
\phi_{\mathbf{t}}=\lambda_{00 \ldots 00} \sum_{\mathbf{d}} \lambda_{\mathbf{d}} \mathrm{x}_{1}^{\mathrm{i}_{1}} \mathrm{x}_{2}^{\mathrm{i}_{2}} \ldots \mathrm{x}_{\mathbf{k}-1}^{\mathrm{i}_{\mathrm{k}-1}} \mathbf{x}_{\mathbf{k}}
$$

From (10), $\phi_{\mathbf{t}_{1} \mathbf{t}_{2} \ldots \mathbf{t}_{\mathbf{k}-1} 0}=\lambda_{00 \ldots 00}$ with multiplicity

$$
\left(n_{k}-1\right) \sum_{t_{1} t_{2} \ldots t_{k-1}=0}^{1} \prod_{r=1}^{k-1}\left(n_{r}-1\right)^{1-t_{r}}=\left(n_{k}-1\right) \prod_{z=1}^{k-1} n_{r} .
$$

So $2^{k-1}$-eigenvalues of (9) are the same and equal to the smallest eigenvalue $\lambda_{00 \ldots .00}$. The corresponding idempotent matrix for $\lambda_{00 \ldots 00}$ will be

$$
\mathbf{M}_{\mathbf{k}-1} \otimes\left(\mathbf{I}_{\mathbf{r}}-\frac{1}{\mathbf{n}_{\mathbf{r}}} \mathbf{J}_{\mathbf{r}}\right)
$$

where a matrix $\mathbf{M}_{\mathrm{k}-1}$ of order $\prod_{r=1}^{k-1} \mathbf{n}_{\mathrm{r}}$ is:

with

$$
\mathbf{M}_{\mathrm{k}-1}=\sum_{\mathbf{t}_{1} \mathbf{t}_{2} \ldots \mathbf{t}_{\mathrm{k}-1}=\mathbf{0}}^{1} \mathbf{P}_{\mathbf{t}_{1}} \otimes \mathbf{P}_{\mathbf{t}_{2}} \otimes \ldots \otimes \mathbf{P}_{\mathbf{t}_{\mathrm{k}}-\mathbf{1}}
$$

$$
\begin{aligned}
\operatorname{rank}\left(\mathbf{M}_{\mathbf{k}-1}\right) & =\sum_{t_{1} t_{2} \ldots t_{k-1}=0}^{1} \operatorname{rank}\left(\mathbf{P}_{\mathbf{t}_{1}}\right) \operatorname{rank}\left(\mathbf{P}_{\mathbf{t}_{2}}\right) \ldots \operatorname{rank}\left(\mathbf{P}_{\mathbf{t}_{\mathrm{k}}-1}\right) \\
& =\sum_{t_{1} \mathbf{t}_{2} \ldots \mathrm{t}_{\mathrm{k}-1}=0}^{1} \prod_{\mathrm{r}=1}^{\mathbf{k}-1}\left(\mathrm{n}_{\mathrm{r}}-1\right)^{1-\mathbf{t}_{\mathrm{r}}}=\prod_{\mathrm{r}=1}^{\mathrm{k}-1} \sum_{\mathrm{t}_{\mathrm{r}}=0}^{1}\left(\mathrm{n}_{\mathrm{k}}-1\right)^{1-\mathbf{t}_{\mathrm{r}}}=\prod_{\mathrm{r}=1}^{\mathrm{k}-1} \mathbf{n}_{\mathrm{r}} .
\end{aligned}
$$

Then $\mathbf{M}_{k-1}$ is an identity matrix since the full rank idempotent matrix is unique and equal to an identity matrix.

The eigenvalue of (9) is of the form

$$
\phi_{t_{1} t_{2} \ldots t_{k-1}}^{*}=\phi_{t_{1} t_{2} \ldots t_{k-1} 1}=\lambda_{00 \ldots 00}+n_{k} \sum_{d} \lambda_{d} x_{t_{1}}^{i_{1}} x_{t_{2}}^{i_{2}} \ldots x_{t_{k-1}}^{i_{k-1}} .
$$

Then the spectral decomposition of (9) according to $2^{k-1}+1$ (possibly) distinct eigenvalues of (9) is:

$$
\begin{aligned}
V & =\lambda_{00 \ldots 00} \mathbf{I}_{12 \ldots \mathrm{k}-1} \otimes\left(\mathbf{I}_{\mathrm{k}}-\frac{1}{\mathbf{n}_{\mathrm{k}}} \mathbf{J}_{\mathbf{k}}\right) \\
& +\sum_{\mathrm{t}_{1} \mathrm{t}_{2} \ldots \mathrm{t}_{\mathrm{k}}-\mathbf{1}}^{1} \phi_{\mathrm{t}_{1} \mathrm{t}_{2} \ldots \mathrm{t}_{\mathrm{k}-\mathrm{k}}}^{*} \mathbf{P}_{\mathrm{t}_{1}} \otimes \mathbf{P}_{\mathrm{t}_{2}} \otimes \ldots \otimes \mathbf{P}_{\mathrm{t}_{\mathrm{k}-1}} \otimes \frac{1}{\mathrm{n}_{\mathbf{k}}} \mathbf{J}_{\mathbf{k}}
\end{aligned}
$$


where $\mathbf{I}_{12 \ldots k-1}$ is an identity matrix of order $\prod_{r=1}^{k-1} n_{r}$ and $P_{t_{r}}$ is in (8).

\section{CONCLUDING REMARKS}

The spectral decomposition of $\mathbf{V}$ provides easily the computation of $\mathbf{V}^{\alpha}$ for any real $\alpha$ since

$$
\mathbf{V}^{\alpha}=\sum_{\mathbf{t}} \phi_{t}^{\alpha} \mathbf{M}_{\mathbf{t}}
$$

where $\phi_{t}$ and $\mathbf{M}_{t}$ are defined in (2) and (5) respectively.

The nonnegative parameters $\lambda_{d}$ in (1) correspond to variance components. From (2), the eigenvalues of the variance-covariance matrix is the linear combination of variance components. It is not necessary to recompute the spectral decomposition of $\mathbf{V}^{*}$ where a new variance-covariance matrix $\mathbf{V}^{*}$ is obtained by removing some $\lambda_{\mathbf{d}}$ 's from $\mathbf{V}$. It can be obtained by removing the corresponding $\lambda_{d}$ 's from the spectral decomposition of $\mathbf{V}$.

A half number of eigenvalues of $\mathbf{V}$ are same and equal to the smallest eigenvalue when there is a replication. In this case, the summations in both (11) and (12) are taken over $2^{\mathrm{k}-1}$, instead of $2^{\mathrm{k}}$. This facilitates the computation of the spectral decomposition of $\mathbf{V}$.

\section{AN EXAMPLE}

Consider the two-way random effect model $y_{i j k}=\mu+a_{i}+b_{j}+c_{i j}+e_{i j k}, i=1,2, \ldots, n_{1}$, $\mathrm{j}=1,2, \ldots, \mathrm{n}_{2}, \mathrm{k}=1,2, \ldots, \mathrm{n}_{3}$ where

$$
a_{i} \sim N\left(0, \sigma_{a}^{2}\right), b_{j} \sim N\left(0, \sigma_{b}^{2}\right), c_{i j} \sim N\left(0, \sigma_{c}^{2}\right), e_{i j k} \sim N\left(0, \sigma_{e}^{2}\right)
$$

and they are independent. The variance-covariance matrix for this model is:

$$
V=\sigma_{\mathrm{a}}^{2} \mathbf{I}_{1} \otimes \mathbf{J}_{2} \otimes \mathbf{J}_{3} \otimes \sigma_{b}^{2} \mathbf{J}_{1} \otimes \mathbf{I}_{2} \otimes \mathbf{J}_{3} \otimes \sigma_{\mathrm{c}}^{2} \mathbf{I}_{1} \otimes \mathbf{I}_{2} \otimes \mathbf{I}_{3} \otimes \sigma_{\mathrm{e}}^{2} \mathbf{I}_{1} \otimes \mathbf{I}_{2} \otimes \mathbf{I}_{3}
$$

where $I_{r}$ and $J_{r}$ for $=1,2,3$ are $a n_{r} \times n_{r}$ identity matrix and $a n_{r} \times n_{r}$ matrix of ones respectively.

Define $\lambda_{000}=\sigma_{e}^{2}, \lambda_{001}=\sigma_{c}^{2}, \lambda_{011}=\sigma_{a}^{2}, \lambda_{101}=\sigma_{b}^{2}$ and the other $\lambda_{d}$ 's are zero. The $2^{3}$ eigenvalues of $\mathbf{V}$ are:

$$
\begin{aligned}
\phi_{t_{1} t_{2} t_{3}} & =\sum_{i_{1} i_{2} i_{3}=0}^{1} \lambda_{i_{1} i_{2} i_{3}} x_{t_{1}}^{i_{1}} x_{t_{2}}^{i_{2}} x_{t_{3}}^{i_{3}} \\
& =\lambda_{000}+\lambda_{001} x_{t_{3}}+\lambda_{011} x_{t_{2}} x_{t_{3}}+\lambda_{101} x_{t_{1}} x_{t_{3}}
\end{aligned}
$$

where $x_{t_{r}}=0$ if $t_{r}=0, x_{t_{r}}=n_{r}$ if $t_{r}=1$ for $r=1,2,3$. Then

$$
\begin{aligned}
& \phi_{000}=\phi_{010}=\phi_{100}=\phi_{110}=\lambda_{000}, \phi_{001}=\lambda_{000}+\mathrm{n}_{3} \lambda_{001}, \phi_{011}=\lambda_{000}+\mathrm{n}_{3} \lambda_{001}+\mathrm{n}_{2} \mathrm{n}_{3} \lambda_{011}, \\
& \phi_{101}=\lambda_{000}+\mathrm{n}_{3} \lambda_{001}+\mathrm{n}_{1} \mathrm{n}_{3} \lambda_{101}, \phi_{111}=\lambda_{000}+\mathrm{n}_{3} \lambda_{001}+\mathrm{n}_{1} \mathrm{n}_{3} \lambda_{101}+\mathrm{n}_{2} \mathrm{n}_{3} \lambda_{011} .
\end{aligned}
$$

The spectral decomposition of $\mathbf{V}$ is: 


$$
\begin{aligned}
V=\phi_{000} I_{12} \otimes\left(I_{3}-\frac{1}{n_{3}} J_{3}\right)+\phi_{001} I_{1} \otimes I_{2} \otimes \frac{1}{n_{3}} J_{3} \\
+\phi_{011}\left(I_{1}-\frac{1}{n_{1}} J_{1}\right) \otimes \frac{1}{n_{2}} J_{2} \otimes \frac{1}{n_{3}} J_{3} \\
+\phi_{101} \frac{1}{n_{1}} J_{1} \otimes\left(I_{2}-\frac{1}{n_{2}} J_{2}\right) \otimes \frac{1}{n_{3}} J_{3} \\
+\phi_{111} \frac{1}{n_{1}} J_{1} \otimes \frac{1}{n_{2}} J_{2} \otimes \frac{1}{n_{3}} J_{3} .
\end{aligned}
$$

\section{Acknowledgment}

The author thanks the referee for making comments which clearify the paper.

\section{REFERENCES}

[1] R. SEARLE, and H. V. HENDERSON. Dispersion Matrices for Variance Components Models, Journal of American Statistical Association, 74. (1979), 465-470

[2] T. WANSBEEK and A. KAPTEYN, A Note on Spectral Decomposition and Maximum Likelihood Estimation in ANOVA Models with Balanced Data, Statistics I\& Probability Letters, 1 (1983), 213-215. 